\title{
Struma ovarii - ultrasound features of a rare tumor mimicking ovarian cancer
}

\author{
Vit Weinberger ${ }^{1}$, Jana Kadlecová1, Luboš Minář́1, Michal Felsinger'1, Milan Anton', \\ Petra Ovesná ${ }^{2}$, Marketa Bednaříková ${ }^{3}$, Marta Č́íhalová ${ }^{4}$, Eva Jandáková ${ }^{4}$, \\ Jitka Hausnerová ${ }^{4}$, Michal Zikán $^{5}$
}

${ }^{1}$ Department of Gynecology and Obstetrics, University Hospital in Brno and Masaryk University, Brno, ${ }^{2}$ Institute of Biostatistics and Analyses, Faculty of Medicine, Masaryk University, Brno, ${ }^{3} \mathrm{Clinic}$ of Internal Medicine-Hematology and Oncology, University Hospital in Brno and Masaryk University, Brno, ${ }^{4}$ Institute of Pathology, University Hospital in Brno and Masaryk University, Brno, ${ }^{5}$ Department of Gynecology and Obstetrics Charles University in Prague - First Faculty of Medicine and Hospital Na Bulovce, Czech Republic

\begin{abstract}
Aims: To describe the ultrasound features of benign struma ovarii that often mimic ovarian cancer in the background of complex clinical and histopathological pictures. Material and methods: We retrospectively identified patients with histologically confirmed benign struma ovarii, treated in our institution between 2003-2016 with complete imaging, clinical, and histopathological data available. Ultrasound findings were drawn from images, and reports using terms and definitions of the International Ovarian Tumor Analysis group and pattern recognition description was applied. Results: In all, 19 patients were identified; 10 with pure and 9 with impure struma. Median age was 47 (range 24-69); 10 (53\%) were premenopausal. Only four $(21 \%)$ patients presented with pain, others were asymptomatic. Using pattern recognition, 74\% strumas (14/19) were uni-/multilocular solid or solid tumors. The solid components were roundish with smooth contours. Six struma pearls were detected. The subjective color score was moderate or abundant in the majority of solid components. Only 5 (26\%) tumors were purely cystic. Conclusions: The ultrasound characteristics differ widely from typical mature ovarian teratoma. Features such as, solid roundish components with smooth contours, struma pearls, acoustic shadowing and occasionally signs of dermoid are clues and may help preoperatively to differentiate benign struma from malignant adnexal lesions.
\end{abstract}

Keywords: dermoid cyst; Doppler; ovary; struma ovarii; ultrasonography

\section{Introduction}

Struma ovarii is a rare ovarian tumor reported to represent up to $1 \%$ of all ovarian tumors and $3 \%$ of all ovarian teratomas [1]. Mature cystic teratoma (dermoid cysts) typically contains mature tissues of ectodermal, mesodermal, and endodermal origin. A struma ovarii is typically composed of follicles filled with eosinophilic

Received 07.04.2018 Accepted 08.06.2018

Med Ultrason

2018, Vol. 20, No 3, 355-361

Corresponding author: Vit Weinberger, M.D., Ph.D.,

Department of Gynecology and Obstetrics,

University Hospital Brno and Masaryk University

Jihlavska 20, Brno, 625 000, Czech Republic

Phone + 420532233843

E-mail: vit.weinberger@gmail.com colloid and lined by a single layer of cuboidal epithelial cells with uniform bland nuclei and eosinophilic cytoplasm. It is defined as a teratoma which contains entirely thyroid tissue (pure struma ovarii) or partially (impure struma ovarii) and is classified as benign or malignant [2]. Macroscopically, struma ovarii is a round or oval tumor of any size, surrounded by a firm capsule and can be detected at any age. Malignancy arising from benign struma ovarii is rare $[3,4]$. Most patients are asymptomatic. A symptomatic tumor may affect adjacent structures and manifest with symptoms related to their compression. Other rare symptoms could be infertility, abnormal uterine bleeding, dysmenorrhea and hot flashes (due to steroid hormone production). Most struma ovarii accumulate iodine and, occasionally (5-20\%), may even produce a significant amount of thyroid hormone. In some 
cases, struma ovarii could be a source of thyrotoxicosis [3]. Benign struma ovarii may be associated with ascites and pleural effusion (pseudo-Meigs' syndrome) [3,4].

There are only few studies aiming to describe ultrasound (US) features of struma ovarii. Zalel et al struggled to find specific features to distinguish between 12 struma ovarii and 32 dermoid cysts on their US appearance [5]. Savelli et al reported 30 patients with benign and 1 with malignant struma ovarii and described struma pearl in $29 \%$ of patients as a possible characteristic sign of struma ovarii [6]. Royo et al reported 9 patients with struma ovarii and concluded that US patterns differ from mature teratomas and that they may be misinterpreted as ovarian cancer in many cases [7].

An appropriate preoperative US examination is crucial in distinguishing between benign and malignant tumors and for further management of ovarian masses. However, despite well-established International Ovarian Tumor Analysis (IOTA) imaging criteria for ovarian cancer recognition, US findings in struma ovarii tumors overlap with those typical of ovarian cancer which contributes to serious difficulties in image interpretation [8]. Therefore, we reviewed US and clinical data for histologically confirmed cases of benign struma ovarii treated in our institution. Our study is complex, related on real clinical data and daily routine practice in our oncologic gynecology center. The aim was to describe the US features (morphological and vascular ultrasound patterns) of struma ovarii and to encourage sonographers dealing with ovarian mass findings to improve their knowledge and awareness of this rare tumor. Correct preoperative diagnosis of struma ovarii as a benign pelvic mass enables the choice of a less radical surgical procedure and minimizes fear in patients who may anticipate an ovarian cancer diagnosis.

\section{Materials and methods}

\section{Study design and patient population}

We conducted a retrospective study of struma ovarii tumors diagnosed and treated in our institution between January 2003 and December 2016. The indications for transvaginal and transabdominal US were prior inconclusive examination with suspicion of gynecological cancer due to a palpable adnexal mass, abnormal US findings assessed by a general gynecologist or general practitioner, with or without elevated CA 125 tumor marker, or nonspecific abdominal symptoms (abdominal/pelvic pain). Most clinical information was retrieved retrospectively from the patient records, such as age at diagnosis, preor postmenopausal status, serum level of CA 125, risk of malignancy index (RMI - combines serum CA 125, menopausal status and US score), symptoms, presence of ascites according to the IOTA consensus described by Timmerman et al [9] and detailed histological evaluation results. Only patients with histologically proven pure or impure struma ovarii were included in the final analysis. Any tumor composed of a dermoid cyst and struma which comprised $>50 \%$ of the tumor volume was defined as an 'impure' struma ovarii. All patients underwent surgery and the tumor was removed. The type of surgery (laparoscopy or laparotomy) and the extension ranging from a simple cystectomy or unilateral salpingo-oophorectomy to more extensively as a total hysterectomy and bilateral salpingo-oophorectomy were reviewed. Frozen section requests were carefully recorded and analyzed.

This study was approved by the local Ethics Committee, and all patients gave their informed consent.

\section{Ultrasound examination}

Each patient underwent a pre-operative US examination according to the methodology and protocol of our institution that consisted of both transabdominal and transvaginal examinations performed within 21 days prior surgery by a gynecologist experienced in US diagnostics in gynecologic oncology. These were expert sonographers with gynecology and oncology experience, certified by the Czech Gynecologic Ultrasound Society as high-level experts in onco-gynecologic sonography. The US examinations were performed in B-mode and color and power Doppler mode with both a transvaginal $(5-8 \mathrm{MHz})$ and a transabdominal probe $(3.5-5 \mathrm{MHz})$ on a variety of equipment. Each US examination was immediately described in the written report and images were stored; both were used in the study analysis. Descriptions and examination reports were performed according to the standards in our center that include the terms and definitions published by the IOTA group [9]. The reports also included the final assessment of the sonographer whether the mass was suspicious for malignancy or not, and the final diagnosis suggested by the sonographer. Results of Doppler examinations were reported in terms of a subjective color score [9].

\section{Statistical analysis}

All clinical and ultrasound parameters were summarized using absolute and relative frequencies or median and range in case of continuous data. All tables were stratified according to histology into patients with pure and impure struma. No statistical comparisons were made because of the descriptive character of the study.

\section{Results}

We retrospectively included 19 patients into the study who had histologically proven benign struma ovarii. No malignant struma ovarii was found. The clinical characteristics of studied patients are detailed in Table I. The 
Table I. Clinical characteristics of patients

\begin{tabular}{llll}
\hline & Pure struma (n=10) & Impure struma (n=9) & Total (n=19) \\
\hline Age (years) & $47(25-67)$ & $42(23-69)$ & $47(23-69)$ \\
Serum CA 125 level (IU/L)] & $20(7-40)$ & $15(10-37)$ & $20(7-40)$ \\
RMI & $65(20-360)$ & $42(15-333)$ & $60(15-360)$ \\
$\quad$ Premenopausal patients & $4(40)$ & $6(67)$ & $10(53)$ \\
$\quad$ Postmenopausal patients & $6(60)$ & $3(33)$ & $9(47)$ \\
Symptomatology & & & \\
$\quad$ Asymptomatic & $7(70)$ & $8(89)$ & $15(79)$ \\
$\quad$ Pelvic pain & $3(30)$ & $1(11)$ & $4(21)$ \\
\hline
\end{tabular}

Values are median (range) or n (\%); RMI - risk of malignancy index

Table II. Diagnosis (benign/uncertain/malignant) according to ultrasound examination

\begin{tabular}{llll}
\hline & Pure struma $(\mathbf{n}=\mathbf{1 0})$ & Impure struma $(\mathbf{n}=9)$ & Total $(\mathbf{n}=\mathbf{1 9})$ \\
\hline Ultrasound diagnosis & & & \\
Benign & $7(70)$ & $6(67)$ & $13(68)$ \\
Uncertain & $0(0)$ & $1(11)$ & $1(5)$ \\
Malignant & $3(30)$ & $2(22)$ & $5(27)$ \\
Specific diagnosis suggested by ultrasound examiner & & & \\
Dermoid & $2(20)$ & $3(33)$ & $5(26)$ \\
Cystadenom/Cystadenofibrom & $4(40)$ & $3(34)$ & $7(38)$ \\
Thecoma & $1(10)$ & $0(0)$ & $1(5)$ \\
Uncertain (not know) & $0(0)$ & $1(11)$ & $1(5)$ \\
Malignant adnexal mass & $3(30)$ & $2(22)$ & $5(26)$ \\
\hline
\end{tabular}

The results are expressed in number (\%); n- number of patients

Table III. Ultrasound characteristics and surgical management of struma ovarii

\begin{tabular}{|c|c|c|c|}
\hline & Pure struma $(n=10)$ & Impure struma $(n=9)$ & Total $(n=19)$ \\
\hline Largest diameter (mm) & $70(27-160)$ & $70(30-200)$ & $70(35-200)$ \\
\hline Largest solid component (mm) & $21.5(0-120)$ & $22.5(0-90)$ & $21.5(0-120)$ \\
\hline \multicolumn{4}{|l|}{ Laterality } \\
\hline Unilateral & $10(100)$ & $9(100)$ & $19(100)$ \\
\hline Bilateral & $0(0)$ & $0(0)$ & $0(0)$ \\
\hline \multicolumn{4}{|l|}{ Type of tumor } \\
\hline Unilocular & $3(30)$ & $0(0)$ & $3(16)$ \\
\hline Unilocular-solid & $1(10)$ & $4(45)$ & $5(26)$ \\
\hline Multilocular & $1(10)$ & $1(11)$ & $2(11)$ \\
\hline Multilocular-solid & $2(20)$ & $3(33)$ & $5(26)$ \\
\hline Solid & $3(30)$ & $1(11)$ & $4(21)$ \\
\hline Struma pearls & $1(10)$ & $5(56)$ & $6(32)$ \\
\hline Acoustic shadowing & $1(10)$ & $4(44)$ & $5(26)$ \\
\hline Ascites & $1(10)$ & $0(0)$ & $1(5)$ \\
\hline \multicolumn{4}{|l|}{ Echogenicity of cyst fluid } \\
\hline Anechogenic & $4(40)$ & $5(56)$ & $9(48)$ \\
\hline Ground glass & $0(0)$ & $1(11)$ & $1(5)$ \\
\hline Low level & $3(30)$ & $2(22)$ & $5(26)$ \\
\hline \multicolumn{4}{|l|}{ Doppler results: Color score } \\
\hline None & $4(40)$ & $1(11)$ & $5(26)$ \\
\hline Minimal & $0(0)$ & $0(0)$ & $0(0)$ \\
\hline Moderate & $2(20)$ & $6(67)$ & $8(42)$ \\
\hline Abundant & $4(40)$ & $2(22)$ & $6(32)$ \\
\hline \multicolumn{4}{|l|}{ Surgery } \\
\hline Frozen section & $3(30)$ & $3(33)$ & $6(32)$ \\
\hline Cystectomy Laparoscopy & $4(40)$ & $2(22)$ & $6(32)$ \\
\hline SO - Laparoscopy & $2(20)$ & $3(33)$ & $5(26)$ \\
\hline SO - Laparotomy & $0(0)$ & $1(12)$ & $1(5)$ \\
\hline $\mathrm{TAH}+\mathrm{BSO}$ & $4(40)$ & $3(33)$ & $7(37)$ \\
\hline
\end{tabular}

Values are median (range) or n (\%). SO - salpingo-oophorectomy, TAH - total abdominal hysterectomy, BSO - bilateral salpingo-oophorectomy 


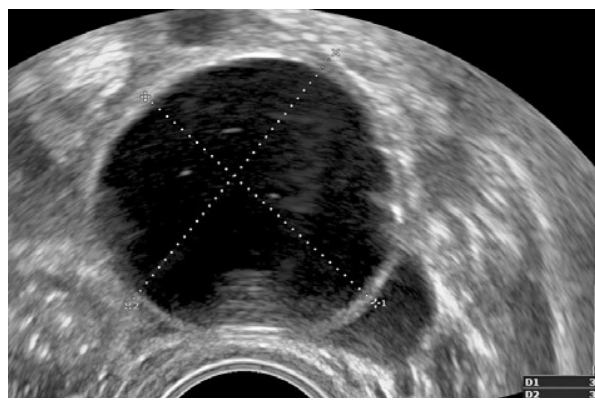

Fig 1. Transvaginal scan. Benign pure struma ovarii - unilocular cyst with anechogenic content. The tumor was classified as benign on preoperative ultrasound examination.

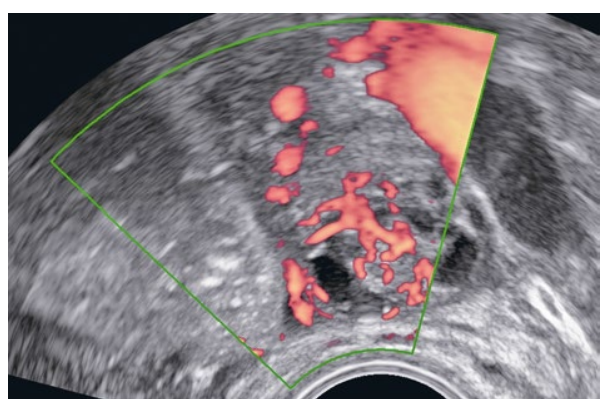

Fig 2. Transvaginal color Doppler image of a pure struma ovarii - centrally located vessels are visible within solid areas, was classified as malignant on preoperative ultrasound examination.

Table IV. Pattern recognition characteristics of struma ovarii

\begin{tabular}{lll}
\hline & Pure struma (n=10) & Impure struma (n=9) \\
\hline Unicolular or multilocular tumor with no specific characteristics* & 4 & 1 \\
Unilocular or multilocular solid tumor with solid vascularized area at Doppler, & 2 & 2 \\
solid component well-circumscribed round with smooth contours & 1 & 5 \\
Tumor with one or more 'struma pearls' & 3 & 1 \\
Solid tumor with moderate/abundant blood flow & 3 & \\
\hline
\end{tabular}

* struma solid structure, pearls, shadowing

diagnosis (benign/uncertain/malignant) suggested by the sonographer and a specific diagnosis for each tumor are presented in Table II. The sonographic characteristics (Bmode and Doppler findings) of pure and impure struma ovarii are described below and in detail together with the surgical management summarized in Table III.

\section{Pure struma ovarii}

There was no uniform US appearance of pure struma ovarii recorded. The median size was $70 \mathrm{~mm}$ (range 37$120)$ and tumors were found only unilaterally. More than half of the cases $(6 / 10,60 \%)$ of pure struma ovarii contained solid components, while only a cystic component was presented in 4 masses $(40 \%)$. The intraluminal fluid was in 4 cases (60\%) anechogenic (fig 1) and in $3(40 \%)$ of low-level echogenicity. Ascites was presented in one patient. All tumors containing solid component were moderately and abundantly vascularized (color score 3 or 4 on a subjective scale). A shadowing of the acoustic signal behind the cyst was observed in one case. Solid components together with the rich vascular supply and absence of shadowing misled an experienced sonographer to describe the tumor as malignant in 3 cases (30\%) and frozen section was performed perioperatively (fig 2). Applying pattern recognition description (Table IV), pure struma ovarii revealed following patterns: 3 strumas were unilocular cysts, and 1 was multilocular without any specific ultrasound characteristics. All these were correctly classified as benign. Three strumas were purely solid, 2 multilocular solid, and 1 unilocular solid. Two tumors contained well-circumscribed round solid areas with smooth contours, and 1 contained struma pearls. Color Doppler revealed moderate or abundant vascularization within all strumas containing solid components intraluminally.

\section{Impure struma ovarii}

There was no uniform sonographic appearance of impure struma documented and did not widely differ from pure stroma ovarii cases. No ascites was noted. The majority $(8 / 9,89 \%)$ of impure struma contained solid components; only one tumor was purely cystic (fig 3). Color Doppler revealed moderate or abundant vascularization within all struma ovarii containing solid components. The cystic fluid was anechogenic in 5 cases (55\%), low-level in $2(22 \%)$, and there was 1 (11\%) case of ground-level echogenicity. Shadowing of the acoustic signal behind the cyst (fig 4) was observed in 4 cases (44\%). Three tumors were described by the sonographer as malignant or uncertain and frozen section was performed intraoperatively. Applying pattern recognition descriptions (Table IV), impure struma ovarii were as follows: one impure struma ovarii was purely multilocular, without any specific ultrasound characteristics. It was correctly classified as benign by the sonographer. One impure struma was purely solid (fig 5), 2 were unilocular or multilocular solid with a well circumscribed solid portion with a rich blood supply at Doppler and 5 $(56 \%)$ contained well vascularized "struma pearls" (fig 6 , fig 7). 


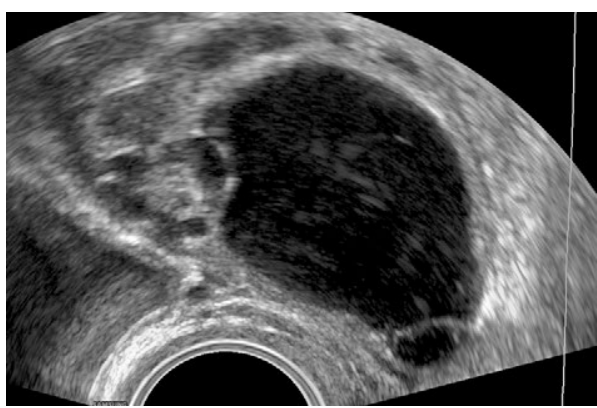

Fig 3. Transvaginal scan. Characteristic B-mode ultrasound findings of impure struma ovarii: a multilocular cystic-solid mass with anechogenic content is visible.

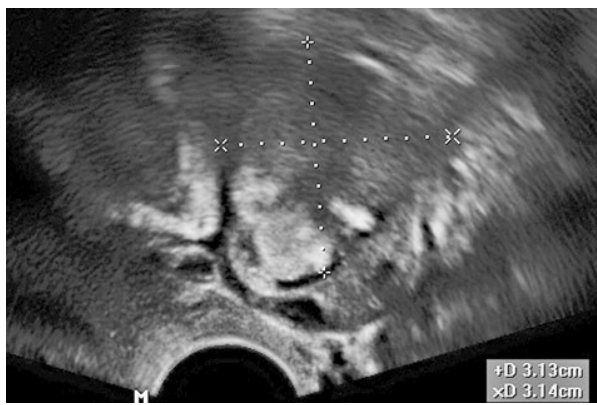

Fig 4. Transvaginal scan. Ultrasound image of benign impure struma ovarii with well circumscribed smooth solid area with strong shadowing behind the tumor. The tumor was classified as benign on preoperative ultrasound examination.

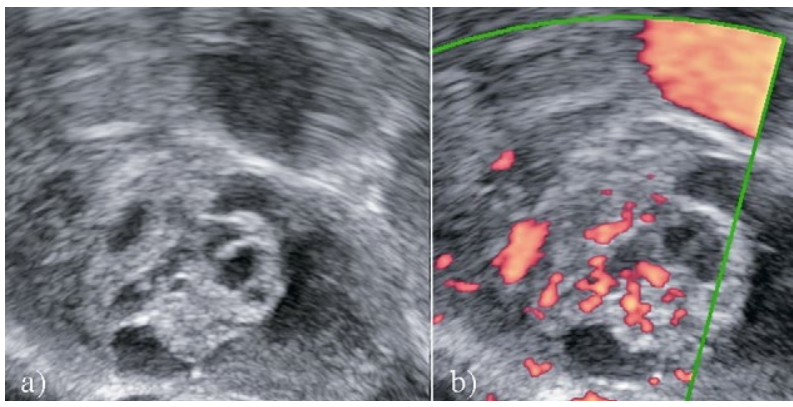

Fig 5. Transvaginal scan. Ultrasound images of benign impure struma ovarii appearing as a solid tumor on ultrasound examination. The gray-scale ultrasound image (a) shows a solid ovarian tumor with irregular internal echogenicity and cystic spaces containing anechogenic cyst fluid. The color Doppler image (b) shows the tumor to be moderately vascularized. The examiner was uncertain whether the tumor was benign or malignant on preoperative ultrasound examination.

\section{Surgical management}

All 19 patients underwent surgery and the ovarian mass was removed: 6 patients were treated with cystectomy, 6 with a salpingo-oophorectomy, and 7 underwent

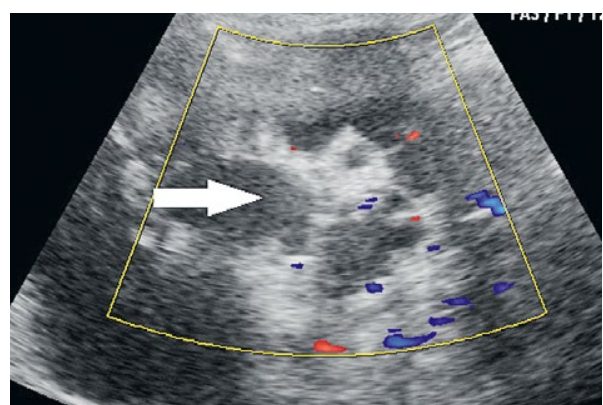

Fig 6. Transvaginal scan. Ultrasound image of benign impure struma ovarii with a typical 'struma pearl' seen on ultrasound examination. The gray-scale ultrasound image shows the 'struma pearl' (arrow).

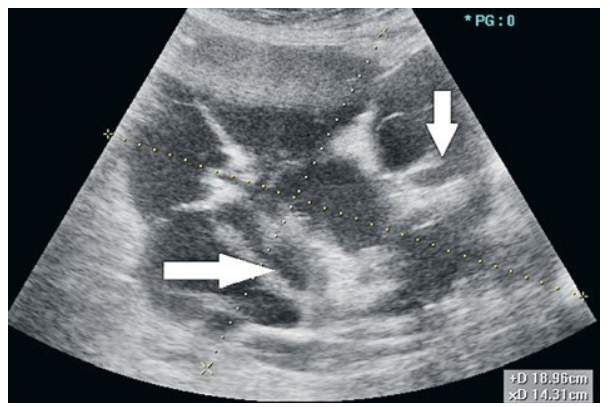

Fig 7. Transvaginal scan. Gray-scale ultrasound image of benign impure struma ovarii with a typical 'struma pearl' (arrows). The tumor was classified as benign on preoperative ultrasound examination.

a total abdominal hysterectomy with bilateral salpingooophorectomy. Surgery was performed via laparoscopy in $11(58 \%)$ cases and via laparotomy in rest of the cases. Six patients underwent surgical staging for ovarian cancer consisting of peritoneal washing, a macroscopic assessment of intraabdominal organs, and preoperative frozen section. These cases were described as suspicious adnexal mass according to the preoperative US examination.

\section{Discussion}

We present a series of 19 patients with benign struma ovarii diagnosed and treated in our institution. Typically, struma ovarii occurs regardless of menopausal status. Most women were asymptomatic and the pelvic mass was an incidental finding with negative tumor markers $[2,3]$. Generally, an adnexal mass and raised tumor marker CA 125 in postmenopausal woman may indicate malignancy. According several well-designed studies, tumor marker CA 125 and RMI are not suitable for distinguishing between benign and malignant adnexal mass [10]. Only 2 patients in our study manifested an elevated 
CA 125 level. One was a patient with simultaneously presented ascites due to the exacerbation of chronic pancreatitis. Six patients had RMI score above 200. In four patients with positive RMI, frozen section was performed because the sonographer preoperatively considered them as malignant.

The diagnosis of mature cystic teratoma at computed tomography (CT) and magnetic resonance imaging (MRI) imaging is straightforward as these modalities are sensitive for fat. Fat is reported in $93 \%$ of cases and teeth or other calcifications in 56\% [11]. US features of commonly diagnosed mature teratomas (dermoids) are well known as an echogenic mass with shadowing behind the tumor caused by sebaceous content and hair inside the cyst cavity, a mural hyperechoic Rokitansky nodule, and echogenic bands intraluminally caused by hair. In dermoid, blood flow can be detected in the capsule only [9]. According to IOTA terminology, the hyperreflective and avascular area in the center of a dermoid cyst should not be classified as a solid component [9]. Struma ovarii picture is different, in most cases no fat is evident. On MRI the most frequent manifestation of struma ovarii is the tumor of multilobulated surface, thickened septa, multiple cysts of variable signal intensities, and enhancing solid components [12]. On CT high attenuation areas and calcifications in the solid components are common findings. On iodine scintigraphy and radioimmunoscintigraphy, struma ovarii reveals a high uptake $[12,13]$. In our study no patient underwent preoperative MRI or CT because these are not a standard imaging method in our institution. US features of struma ovarii are not similar to those of mature cystic teratomas and differ widely both in gray scale and Doppler imaging [6,7]. As an evidence of this, in our study 14 tumors (74\%) had a complex appearance (solid component within the tumor present) in ultrasound examination. Four tumors were purely solid and 4 had well-circumscribed smooth solid areas; all solid components were well-vascularized at Doppler. We retrospectively recognized $6(32 \%)$ tumors containing "struma pearls" which were first described by Savelli et al in 2008 [6]. Struma pearl is a solid hyperechoic area centrally vascularized at Doppler examination and resembling a round white ball described in most mature teratomas [14]. Savelli reported this feature as a putative marker and sign of struma ovarii and described this sign in $9 / 31$ cases $(29 \%)$ [6]. We described acoustic shadowing in $26 \%$ of struma ovarii.

We misclassified 6 cases of struma ovarii $(32 \%)$ as malignant because a multilocular solid appearance with central vascularization pattern is typically seen in ovarian cancer patients $[9,15]$. Ultrasound features of struma ovarii misclassified as ovarian cancer tumors were: uni- lateral lesions of median size $95 \mathrm{~mm}$ (range 70-200), unilocular/multilocular solid or solid tumor with moderate or abundant blood flow perfusion at Doppler in solid components or solid mass; no shadowing was visible behind the tumor. This is in concordance with the first phase IOTA study, which reported struma ovarii are commonly misclassified as malignant by experienced sonographers [8].

Conservative laparoscopic surgery is strongly advised for women with a desire for fertility $[16,17]$. There may be a general objection that the laparoscopic removal of a potentially malignant adnexal tumor increases the possibility of tumor spillage and port site metastasis development. Based on our experience, a laparoscopic approach can be easily adopted not only for fertile-age patients but also for most postmenopausal patients. In our series, the tumor was inserted safely into the endobag which enabled extraction from the abdomen without spilling potentially malignant cells into the abdominal cavity. Based on preoperatively suspicious ultrasound results, in 6 patients a frozen section was intraoperatively performed. As evidenced in our series, $90 \%$ of fertile-age patients underwent conservative surgical treatment via laparoscopy for fertility-preservation; just one 42-year-old patient underwent a hysterectomy with bilateral salpingo-oophorectomy at her own request.

We admit several limitations of our study. Firstly, this is a single institution retrospective study. Struma ovarii is a rare tumor, therefore it was necessary to collect cases over a long-time frame. Ultrasound data and examinations were gained by using several ultrasound devices. Although our series of 19 patients with benign struma ovarii seems to be one of the largest reports dealing with complex clinical data, ultrasound reports and descriptions, the number of cases remains small. Therefore, ultrasound features and characteristics of struma ovarii may be biased. The strength of our study is based on a comprehensive unique database of clinical, surgical and pathological data connected with highly experienced preoperative ultrasound performance. Our data represent real clinical life difficulties arising from the ultrasound findings of such strange adnexal mass. Due to the rareness of struma ovarii, more ultrasound and clinical data are required, and our study may contribute to better insights into the proper and adequate diagnostic of this rare ovarian tumor.

\section{Conclusion}

US appearance of struma ovarii varies and its low incidence makes US diagnosis very challenging. The complexity of the ultrasound picture consisting of well- 
vascularized solid components with smooth contours and features such as shadowing, struma pearls, and some US signs of mature teratoma at least partially visible in the tumor, may help even experienced sonographers when deciding whether a lesion is benign or malignant. For fertile-age patients, a laparoscopic surgical approach should be considered and preferred. We believe our study may contribute to the awareness of struma ovarii and thus decrease patients' fear of ovarian cancer and minimize the number of unnecessary laparotomies and extensive surgical procedures.

\section{Acknowledgments}

This work was supported by the Ministry of Health, Czech Republic - conceptual development of research organization (FNBr 65269705) and grant project No. 1732030A. No assistance was used in the writing of this article.

\section{Conflict of interest: none}

\section{References}

1. Talerman A. Germ cell tumors of the ovary. Curr Opin Obstet Gynecol 1997;9:44-47.

2. Roth LM, Talerman A. The enigma of struma ovarii. Pathology 2007;39:139-146.

3. Talerman A. Germ cell tumors of the ovary. In: Kurman RJ (ed.). Blaustein's Pathology of the Female Genital Tract (5rd edn), New York: Springer Verlag; 2001:967-1035.

4. Ayhan A, Yanik F, Tuncer R, Tuncer ZS, Ruacan S. Struma ovarii. Int J Gynaecol Obstet 1993;42:143-146.

5. Zalel Y, Seidman DS, Oren M, et al. Sonographic and clinical characteristics of struma ovarii. J Ultrasound Med 2000;19:857-861.

6. Savelli L, Testa AC, Timmerman D, Paladini D, Ljungberg $\mathrm{O}$, Valentin L. Imaging of gynecological disease (4): clini- cal and ultrasound characteristics of struma ovarii. Ultrasound Obstet Gynecol 2008;32:210-219.

7. Royo P, Alcazar JL, Virgen M, Mazaira J, Jurado M, Lopez G. B-mode and Doppler features of struma ovarii. Ultrasound Obstet Gynecol 2008;31:109-110.

8. Valentin L, Ameye L, Jurkovic D, et al. Which extrauterine pelvic masses are difficult to correctly classify as benign or malignant on the basis of ultrasound findings and is there a way of making a correct diagnosis? Ultrasound Obstet Gynecol 2006;27:438-444.

9. Timmerman D, Valentin L, Bourne TH, et al. Terms, definitions and measurements to describe the sonographic features of adnexal tumors: a consensus opinion from the International Ovarian Tumor Analysis (IOTA) Group. U1trasound Obstet Gynecol 2000;16:500-505.

10. Valentin L, Jurkovic D, Van Calster B, et al. Adding a single CA 125 measurement to ultrasound imaging performed by an experienced examiner does not improve preoperative discrimination between benign and malignant adnexal masses. Ultrasound Obstet Gynecol 2009;34:345-354.

11. Outwater EK, Siegelman ES, Hunt JL. Ovarian teratomas: tumor types and imaging characteristics. Radiographics 2001;21:475-490.

12. Ikeuchi T, Koyama T, Tamai K, et al. CT and MR features of struma ovarii. Abdom Imaging 2012;37:904-910.

13. Dujardin MI, Sekhri P, Turnbull LW. Struma ovarii: role of imaging? Insights Imaging 2014;5:41-51.

14. Caspi B, Appelman Z, Rabinerson D, Elchalal U, Zalel Y, Katz Z. Pathognomonic echo patterns of benign cystic teratomas of the ovary: classification, incidence and accuracy rate of sonographic diagnosis. Ultrasound Obstet Gynecol 1996; 7:275-279.

15. Yang Q, Yang X, Liu ZZ, et al. Sonographic and Pathologic Features of Struma Ovarii. Zhongguo Yi Xue Ke Xue Yuan Xue Bao 2015;37:309-314.

16. Wee JY, Li X, Chern BS, Chua IS. Struma ovarii: management and follow-up of a rare ovarian tumour. Singapore Med J 2015;56:35-39.

17. Medeiros LR, Rosa DD, Bozzetti MC, et al. Laparoscopy versus laparotomy for benign ovarian tumour. Cochrane Database Syst Rev 2009;(2):CD004751. 